\title{
Heterodera schachtii Nematodes Interfere with Aphid-Plant Relations on Brassica oleracea
}

\author{
W. H. Gera Hol • Wietse De Boer • Aad J. Termorshuizen • \\ Katrin M. Meyer • Johannes H. M. Schneider • \\ Wim H. Van Der Putten • Nicole M. Van Dam
}

Received: 19 April 2013 /Revised: 25 July 2013 / Accepted: 5 August 2013 / Published online: 8 September 2013

(C) The Author(s) 2013. This article is published with open access at Springerlink.com

\begin{abstract}
Aboveground and belowground herbivore species modify plant defense responses differently. Simultaneous attack can lead to non-additive effects on primary and secondary metabolite composition in roots and shoots. We previously found that aphid (Brevicoryne brassicae) population growth on Brassica oleracea was reduced on plants that were infested with nematodes (Heterodera schachtii) prior (4 weeks) to aphid infestation. Here, we examined how infection with root-feeding nematodes affected primary and secondary metabolites in the host plant and whether this could explain the increase in aphid doubling time from 3.8 to 6.7 days. We hypothesized that the effects of herbivores on plant metabolites would depend on the presence of the other herbivore and that nematode-induced changes in primary metabolites would correlate with reduced aphid performance. Total glucosinolate concentration in the leaves was not affected by nematode presence, but the
\end{abstract}

Electronic supplementary material The online version of this article (doi:10.1007/s10886-013-0338-4) contains supplementary material, which is available to authorized users.

W. H. G. Hol $(\bowtie) \cdot$ W. H. Van Der Putten

Department of Terrestrial Ecology, Netherlands Institute of Ecology (NIOO-KNAW), Droevendaalsesteeg 10, 6708 PB Wageningen,

The Netherlands

e-mail: g.hol@nioo.knaw.nl

W. De Boer

Department of Microbial Ecology, Netherlands Institute of Ecology (NIOO-KNAW), Droevendaalsesteeg 10, 6708 PB Wageningen,

The Netherlands

W. De Boer

Department of Soil Quality, Wageningen University,

Droevendaalsesteeg 4, 6708 PB Wageningen, The Netherlands

A. J. Termorshuizen

BLGG Research, Binnenhaven 5, 6709 PD Wageningen,

The Netherlands composition of glucosinolates shifted, as gluconapin concentrations were reduced, while gluconapoleiferin concentrations increased in plants exposed to nematodes. Aphid presence increased 4-methoxyglucobrassicin concentrations in leaves, which correlated positively with the number of aphids per plant. Nematodes decreased amino acid and sugar concentrations in the phloem. Aphid population doubling time correlated negatively with amino acids and glucosinolate levels in leaves, whereas these correlations were non-significant when nematodes were present. In conclusion, the effects of an herbivore on plant metabolites were independent of the presence of another herbivore. Nematode presence reduced aphid population growth and disturbed feeding relations between plants and aphids.

Keywords Heterodera B Brevicoryne - Brassica . Glucosinolates $\cdot$ Phloem $\cdot$ EDTA

K. M. Meyer

Department of Ecosystem Modelling, Georg-August-University of Göttingen, Büsgenweg 4, 37077 Göttingen, Germany

J. H. M. Schneider

IRS Institute of Sugar Beet Research, Van Konijnenburgweg 24, 4600 AA Bergen op Zoom, The Netherlands

W. H. Van Der Putten

Laboratory of Nematology, Wageningen University,

Droevendaalsesteeg 1, 6708 PB Wageningen, The Netherlands

N. M. Van Dam

Department of Ecogenomics, Radboud University Nijmegen, Institute for Water and Wetland Research, Heyendaalseweg 135, 6525 AJ Nijmegen, The Netherlands 


\section{Introduction}

When a plant is attacked simultaneously by different species of the same feeding guild (e.g., chewers, miners, borers, or suckers), it is likely that these herbivores are competing for the same resources. The herbivores can affect each other via direct competition for primary compounds, if both parties tap from the same pool of solutes, or by systemically triggering the plant's defense system (Salt et al. 1996). This is well-studied for herbivores sharing the same shoot, and also increasingly so for spatially separated species such as aboveground and belowground herbivores (Bezemer and van Dam 2005; van Dam and Heil 2011). Interactions between plant-feeding nematodes and aphids are an interesting model to study multiple attackers on one plant since these organisms have remarkable similarities in feeding strategies and salivary composition (Carolan et al. 2011), are sensitive to the same plant resistance genes (Rossi et al. 1998), and often are economically important pests on the same host plant. However, effects of nematodes and aphids on each other might be asymmetric, as there is more evidence of nematodes affecting aphids (e.g., Bezemer et al. 2005; Kaplan et al. 2011; Wurst and van der Putten 2007) than on aphids affecting nematodes (Kutyniok and Müller 2012; Wardle et al. 2004)

The mechanism behind the asymmetric competition between aphids and nematodes may be found in changes in plant biomass or quality. Primary and secondary metabolites are more likely candidates to reduce aphid population growth than simple changes in plant biomass, since nematode presence also can reduce aphids without significantly affecting plant biomass (Vandegehuchte et al. 2010; Wurst and van der Putten 2007). The nature of these changes in plant quality remains unclear, but the involvement of both primary and secondary metabolites has been implied in many studies. Bezemer et al. (2005) found that nematode presence reduced foliar phenolics and amino acids in the phloem, and that nematode presence reduced aphid reproduction. Kaplan et al. (2011) explicitly tested whether the strength of aphid-induced sinks was dependent on the presence of the gall-inducing nematode Meloidogyne incognita (southern root-knot nematode), but found no evidence for competition for primary compounds.

Heterodera spp. are sedentary nematodes like Meloidogyne spp.: both species are vascular feeders inducing changes in plant tissue that redirect photoassimilates from the phloem into their feeding structures (Hofmann et al. 2009). There are few studies on Heterodera-aphid interactions, mostly done with soybean. In those studies, the presence of aphids and nematodes has additive effects on soybean yield (Hong et al. 2010, 2011; McCarville et al. 2012). Alate aphids preferred plants without nematodes over nematode-infested plants (Hong et al. 2010), but a mechanistic explanation is lacking. Hence, plant-mediated interactions between nematodes and aphids are quite common, but the chemical mechanisms are as yet unknown. Only a subset of the nematode-aphid studies has investigated involvement of primary or secondary compounds (e.g. Kutyniok and Müller 2012), while it is known that nematode presence also can affect aboveground levels of secondary compounds (Kaplan et al. 2008; van Dam et al. 2005).

Here, we studied Heterodera-aphid interactions in Brassica oleracea (Brussels sprouts), a plant species with a relatively well-known defense system. Previously, we found that the presence of nematodes ( $H$. schachtii, beet cyst nematode) in the soil severely hampered aphid population growth on $B$. oleracea (Hol et al. 2010). Those data were collected from an experiment where we manipulated soil microbial composition by inoculating a dilution series of soil samples to test the role of rare soil microbes on plant growth and how this could affect plant-herbivore interactions. The effects of variations in soil microbial communities have been published (Hol et al. 2010), while here we present changes in plant metabolites that might explain the effects of nematode presence on aphids. The best known defense compounds of the Brassicaceae are glucosinolates, which are expressed both constitutively and after induction (Hopkins et al. 2009; Textor and Gershenzon 2009). Glucosinolates in Brassica species can be induced by pathogens (Ludwig-Muller et al. 2002; Vierheilig et al. 2000), insects and nematodes (Lohmann et al. 2009; van Dam et al. 2005; Wurst et al. 2006). Nematodes can have species-specific effects on glucosinolate levels in Sinapis alba (white mustard) (Lohmann et al. 2009), with interactions between nematode and earthworm presence. In this study, $M$. incognita increased aromatic glucosinolates only when earthworms were present. Similar results were found by Wurst et al. (2006) showing that Meloidogyne infestation increases glucoraphanin concentrations but only in the presence of earthworms. Another interesting interaction between nematodes and other organisms was found for Pratylenchus sp. (root lesion nematode), which initially lowered glucosinolate concentrations, but primed plants to increase glucosinolate levels quickly after attack by aboveground caterpillars (van Dam et al. 2005). Thus, nematodes affect glucosinolate production in Brassica spp. shoots, and these effects can interact with those from other organisms. However, this will depend on the duration of infestation, as early nematode infection did not affect primary and secondary metabolic profile in Arabidopsis nor aphid population growth (Kutyniok and Müller 2012).

In the present study, we used the aphid Brevicoryne brassicae (cabbage aphid), a specialist that down-regulates production of jasmonic acid (Soler et al. 2012), a signaling molecule involved in inducing plant defense (Mueller et al. 1993). Glucosinolate biosynthesis can be induced by $B$. brassicae, with stronger effects on indole glucosinolates than on aliphatic glucosinolates (Bidart-Bouzat and Kliebenstein 2011; Khan et al. 2011). A positive correlation was found between aphid performance and concentration of aliphatic 
glucosinolates in the phloem (Kos et al. 2012). The potential sensitivity of B. brassicae to glucosinolates (Kos et al. 2012) and the ability of nematodes to induce glucosinolates make changes in the levels of these secondary metabolites a plausible mechanism for explaining negative effects of nematode presence on aphids. However, nematode presence has been found to reduce aphid population growth in many different plant hosts that do not contain glucosinolates, and thus a universal mechanism involving primary metabolites may be involved as well. This could be amino acid concentrations in the leaves (van Emden and Bashford 1971) and phloem (Cole 1997), which are known to correlate with $B$. brassicae performance.

Here, both primary and secondary metabolites were investigated to identify changes in plant biomass and metabolites due to nematode presence, and to link these parameters to the reduction in aphid numbers on $B$. oleracea. Our first hypothesis was that the effects of both herbivores on plant parameters will be non-additive, i.e., effects of one herbivore will be magnified or reduced by the presence of the other. This is based on previous studies that found statistically significant interactions between the presence of nematodes and other organisms with respect to plant secondary metabolites (Lohmann et al. 2009; van Dam et al. 2005; Wurst et al. 2006). Our second hypothesis was that nematode-induced changes in primary metabolites correlate with reduced aphid performance. This is based on the fact that effects of nematode presence on aphids have been found in many plant species and a universal mechanism involving changes in primary metabolites seems more likely than species-specific changes in secondary metabolites.

\section{Methods and Materials}

Experimental Design The experimental setup was designed as a randomized complete block design with nematode (absence/ presence) and aphid (absence/presence) treatments, 7 replicates and 3 soil treatments (Hol et al. 2010). The three soil treatments consisted of increasing dilutions of soil suspensions, which were added to sterilized soil to investigate the effects of bacterial species loss on plant growth (Hol et al. 2010). We used the data from that experiment to investigate plant parameters in relation to nematode presence and aphid performance. Pots filled with $550 \mathrm{~g}$ soil were each sown with 2 seeds of Brassica oleracea 'Maximus' (Syngenta Seeds, Enkhuizen, the Netherlands). Pots were placed randomly on a table in a greenhouse with controlled conditions $\left(60 \% \mathrm{RH} ; 16 \mathrm{~h} \mathrm{~L}, 8 \mathrm{~h} \mathrm{D}, 21^{\circ} \mathrm{C} / 16^{\circ} \mathrm{C}\right)$ and additional lighting. Plants were watered and fertilized regularly (Hol et al. 2010). After 6 week, seedlings were inoculated with 500 juveniles of Heterodera schachtii, suspended in water. This initial density of nematodes is low compared with the advised threshold for growing resistant cultivars in the field (Heijbroek et al. 2002) and compared with the thousands of eggs and juveniles that can be found in $100 \mathrm{~g}$ infested soil (Hbirkou et al. 2011). The juveniles originated from a culture on sugar beets in the greenhouse. Non-nematode treatments received the same amount of water $(5 \mathrm{ml})$, but without nematodes. Four weeks after nematode inoculation, five 4th instar Brevicoryne brassicae were placed on the $B$. oleracea plants that were allocated to aphid treatment. All pots were placed individually inside a fine-maze net cage. Numbers of aphids per plant were counted three times per week, while nematodes were counted only at the end of the experiment. Nematode cysts were extracted from $550 \mathrm{ml}$ soil via stirring/decantation (Hol et al. 2010) and separated from organic debris using the acetone method (Den Ouden 1954). The number of cysts per sample was determined with image analysis software WINSEEDLE PRO v.7 (Regent Instruments, Canada). The average number of larvae and eggs inside the cysts was $195 \pm 14$ ( $\min =20$, $\max =$ $396, N=44$ ). Plants were harvested after a growing period of 16 week. All plants were still in the vegetative stage at harvest. The harvesting took 2 days, 4 blocks were harvested on day 1 and 3 blocks on day 2 . Within blocks, the differences in harvest time for nematode absence/presence was on average less than $4 \mathrm{~min}$, and for aphid absence/presence less than $7 \mathrm{~min}$. Plant shoots and roots were weighed to determine fresh weight, freeze-dried, and weighed again to determine plant dry weight. From each plant, the first two young fully expanded leaves were flash-frozen in liquid nitrogen. The flash-frozen leaves were freeze-dried and ground in a ball mill (Retsch, Germany), and this material was used for analysis of carbon, nitrogen, amino acids, sugars, and glucosinolates.

Organic carbon and total nitrogen were measured in $3 \mathrm{mg}$ of the leaves and roots with a Flash EA $1112 \mathrm{NC}$ analyzer (Interscience, Breda, The Netherlands). Nitrogen allocation to the shoot was calculated on the basis of dry weight as follows: (nitrogen concentration in shoot $\mathrm{x}$ shoot biomass)/(nitrogen concentration in shoot $\mathrm{x}$ shoot biomass + nitrogen concentration in root $\mathrm{x}$ root biomass). Extracts were made from $100 \mathrm{mg}$ ground material to quantify amino acids, sugars, and glucosinolates with an HPLC. Methanol extracts were made according to van Dam et al. (2004), and this extract was used for sugar and amino acid measurements. The extract was diluted 1:100 for sugar and 1:50 for amino acids measurements as described in van Dam and Oomen (2008). Briefly, the diluted sugar extract was run on a Dionex HLPC using a $10 \mathrm{ppm}$ solution containing sorbitol, manitol, trehalose, sucrose, melibiose, glucose, and fructose as references, detection limit $0.1 \mathrm{ppm}$. This reference solution was diluted to $7.5,5$ and $2.5 \mathrm{ppm}$ to obtain a reference curve, and every 10 samples an additional reference sample was injected to check for deviations (van Dam and Oomen 2008). The analyses of amino acids also were done according to van Dam and Oomen (2008), and here the Sigma AA-S-18 amino acid standard (Sigma, St Louis, MO, USA) was used as reference and diluted for calibration with a detection limit of $0.1 \mu \mathrm{mol}$. 
Glucosinolate detection was based on van Dam et al. (2004), using a single wavelength detector set to $226 \mathrm{~nm}$. Sinigrin was used as external standard for the glucinolates, and correction factors from Buchner (1987) were used. Detection limits for glucosinolates were $2 \mu \mathrm{mol}$. A list of detected glucosinolates is given in Table 1. While the first two young fully expanded leafs were freeze-dried and used for glucosinolate analysis, the next fully expanded leaf was used for analysis of phloem by obtaining EDTA leachate (Bezemer et al. 2005): the fully expanded leaf was cut at the petiole and placed in an Eppendorf tube with ultrapure water for $5 \mathrm{~min}$. In order to reduce the contamination with mesophyll, the leaf was transferred after $5 \mathrm{~min}$. to an $2 \mathrm{ml}$ Eppendorf tube with $1.5 \mathrm{ml}$ EDTA $8 \mathrm{mM}, \mathrm{pH}=8$. Later, this solution was analyzed as representing the phloem, but it might have been contaminated with leaf sap. Determination of phloem sap composition via stylectomy would have been more accurate, but exudation with EDTA can be used for rough analyses (Girousse et al. 1991). The leaf was incubated in the dark at room temperature for $2 \mathrm{~h}$ and afterwards the Eppendorf tubes were stored at $-20{ }^{\circ} \mathrm{C}$. EDTA extracts were filtered $(0.2 \mu \mathrm{m})$ and $100 \mu \mathrm{l}$ were used for amino acids and sugar analysis on the HPLC. The total amount of EDTA solution in one Eppendorf was $1.5 \mathrm{ml}$, and the assumption was that the phloem contributed little to this total volume. Thus, the concentrations in the $100 \mu \mathrm{l}$ were used to calculate the total amount of sugars and amino acids in the Eppendorf with $1.5 \mathrm{ml}$, and then divided by the leaf dry weight to correct for initial size differences. The remaining EDTA extract was used for glucosinolate analysis according to van Dam et al. (2004), but omitting the boiling step and directly starting with adding the extract to a DEAESephadex A25 column. Since glucosinolate concentrations in the phloem are relatively low, we selected a subset of the EDTA extracts for analysis on basis of relatively high sugar and amino acid content ( 4 of the 7 blocks in total, $N=48$ ).
After obtaining the EDTA leachate, the leaf was freeze-dried and its weight was determined.

Statistical Analyses The experiment consisted of 84 pots, based on four herbivore treatments (no herbivores, nematodes only, aphids only, nematodes and aphids), seven replicates and a soil microbial treatment with three levels. The replicates were distributed randomly over 7 blocks (fully randomized block design) to account for spatial effects in the greenhouse. The three different soil microbial inoculum treatments where different dilutions of soil suspensions were added have been described previously (Hol et al. 2010). For all parameters presented here, we tested for 3-way interactions between nematodes, aphids, and microbes. The absence of significant 3-way interactions showed that there were no effects of microbial soil treatment on aphid-nematode interactions. Hence, the data shown here were pooled for all three soil treatments, resulting in $N=21$ per herbivore treatment. For the analysis of aphid and nematode effects on plant parameters, a linear mixed effects model (lme) was used with the soil microbial inoculum treatment nested within block as random factor and the presence of nematode and aphids as categorical factors. For all variables (except \%water) presented in Table 2 that were significantly affected by either nematodes or aphids, we verified that there was no significant interaction with soil microbial treatment when this was included as fixed factor. Water percentages showed a significant interaction between nematodes and soil microbial treatment, but ignoring this interaction still gave overall significant main effects of nematodes on moisture. The aphid $\times$ nematode interactions were not significant for any parameters in Table 2, and hence were removed from the model. Most variables were log-transformed to obtain normality of errors, which was assessed by visual inspection of q-q plots. When transformation did not result in sufficiently normal error distributions, non-parametric tests were performed to
Table 1 Abbreviation and Full Names of the 11 Detected Glucosinolates

\begin{tabular}{lll}
\hline Abbreviation & Common name & Synonyms \\
\hline IBE & Glucoiberin & 3-methylsulfinylpropylglucosinolate \\
PRO & Progoitrin & 2(S)-hydroxy-3-butenylglucosinolate \\
RAPH & Glucoraphanin & 4-methylsulphinylbutylglucosinolate \\
SIN & Sinigrin & 2-propenylglucosinolate \\
GNA & Gluconapin & 3-butenylglucosinolate \\
GNL & Gluconapoleiferin & 2-hydroxy-4-pentenylglucosinolate \\
TROP & Glucotropaeolin & Benzylglucosinolate \\
$4 \mathrm{OH}$ & 4-hydroxyglucobrassicin & 4-hydroxy-3-indolylmethylglucosinolate \\
GBC & Glucobrassicin & 3-indolylmethylglucosinolate \\
$4-\mathrm{MeOH}$ GBC & 4-methoxyglucobrassicin & 4-methoxy-3-indolylmethylglucosinolate \\
$\mathrm{NEO}^{\mathrm{a}}$ & Neoglucobrassicin & 1-methoxy-3-indolylmethylglucosinolate \\
\hline
\end{tabular}


Table 2 Mean $( \pm$ Se) of Plant Parameters from Brassica oleracea Plants without Herbivores (Control), with Aphids, Nematodes (Nema), or Both

\begin{tabular}{|c|c|c|c|c|c|c|}
\hline & Control & Aphids & Nema & Aphids +Nema & $P$ - Aphids ${ }^{\mathrm{a}}$ & $P$ - Nema $^{\mathrm{a}}$ \\
\hline Shoot biomass ${ }^{\mathrm{b}}$ & $3.79 \pm 0.13$ & $3.49 \pm 0.17$ & $3.07 \pm 0.13$ & $3.02 \pm 0.11$ & 0.15 & $<0.01$ \\
\hline Root biomass $^{\mathrm{b}}$ & $1.84 \pm 0.11$ & $1.70 \pm 0.07$ & $1.73 \pm 0.11$ & $1.90 \pm 0.14$ & 0.94 & 0.88 \\
\hline Water $\%$ shoot $^{\mathrm{c}}$ & $79.9 \pm 0.3$ & $80.4 \pm 0.4$ & $78.5 \pm 0.3$ & $79.0 \pm 0.4$ & 0.18 & $<0.01$ \\
\hline $\mathrm{N} \%$ shoot & $1.02 \pm 0.04$ & $1.08 \pm 0.07$ & $0.84 \pm 0.05$ & $0.82 \pm 0.03$ & 0.62 & $<0.01$ \\
\hline $\mathrm{N} \%$ root & $1.26 \pm 0.07$ & $1.25 \pm 0.06$ & $1.43 \pm 0.07$ & $1.47 \pm 0.07$ & 0.77 & 0.01 \\
\hline $\mathrm{N}$ shoot allocation ${ }^{\mathrm{d}}$ & $0.63 \pm 0.01$ & $0.63 \pm 0.02$ & $0.51 \pm 0.02$ & $0.48 \pm 0.02$ & 0.48 & $<0.01$ \\
\hline Carbon shoot ${ }^{\mathrm{c}}$ & $41.0 \pm 0.3$ & $40.4 \pm 0.4$ & $41.3 \pm 0.3$ & $40.3 \pm 0.3$ & 0.01 & 0.87 \\
\hline Carbon root ${ }^{\mathrm{c}}$ & $31.0 \pm 1.65$ & $31.2 \pm 1.54$ & $31.7 \pm 1.56$ & $31.5 \pm 1.7$ & 0.99 & 0.72 \\
\hline $\mathrm{C}: \mathrm{N}$ shoot & $41.7 \pm 1.68$ & $39.6 \pm 1.9$ & $52.3 \pm 2.7$ & $50.5 \pm 2.0$ & 0.35 & $<0.01$ \\
\hline $\mathrm{C}: \mathrm{N}$ root & $24.6 \pm 0.57$ & $24.8 \pm 0.45$ & $22.2 \pm 0.33$ & $21.4 \pm 0.5$ & 0.35 & $<0.01$ \\
\hline \multicolumn{7}{|l|}{ Sugars ${ }^{\mathrm{e}}$ Leaf } \\
\hline Total & $46.1 \pm 3.2$ & $43.3 \pm 2.9$ & $49.8 \pm 2.0$ & $50.4 \pm 2.0$ & 0.57 & $<0.01$ \\
\hline Glucose & $23.6 \pm 2.0$ & $21.3 \pm 1.6$ & $25.7 \pm 1.1$ & $26.2 \pm 1.1$ & 0.42 & $<0.01$ \\
\hline Fructose & $16.8 \pm 1.1$ & $16.1 \pm 1.1$ & $18.4 \pm 0.9$ & $18.3 \pm 0.8$ & 0.62 & 0.02 \\
\hline Sucrose & $5.7 \pm 0.4$ & $6.0 \pm 0.5$ & $5.7 \pm 0.3$ & $5.8 \pm 0.3$ & 0.51 & 0.77 \\
\hline \multicolumn{7}{|l|}{ Sugars $^{\mathrm{e}}$ Phloem } \\
\hline Total & $1.63 \pm 0.18$ & $1.81 \pm 0.25$ & $1.31 \pm 0.21$ & $1.15 \pm 0.13$ & 0.66 & $<0.01$ \\
\hline \multicolumn{7}{|l|}{ Amino acids ${ }^{\mathrm{e}}$ Leaf } \\
\hline Total & $92.2 \pm 5.9$ & $89.8 \pm 5.8$ & $95.3 \pm 4.7$ & $94.2 \pm 4.2$ & 0.63 & 0.33 \\
\hline Arginine & $8.1 \pm 0.7$ & $7.9 \pm 0.6$ & $6.3 \pm 0.5$ & $6.1 \pm 0.2$ & 0.67 & $<0.01$ \\
\hline Leucine & $20 . \pm 01.3$ & $21.0 \pm 1.8$ & $19.5 \pm 1.1$ & $19.5 \pm 1.1$ & 0.94 & 0.63 \\
\hline Threonine & $31.2 \pm 2.5$ & $28.7 \pm 2.1$ & $33.8 \pm 1.6$ & $33.6 \pm 1.5$ & 0.40 & 0.02 \\
\hline Tryptophan & $0.6 \pm 0.1$ & $0.7 \pm 0.1$ & $0.7 \pm 0.0$ & $0.8 \pm 0.1$ & 0.04 & 0.02 \\
\hline Valine & $32.4 \pm 2.2$ & $31.6 \pm 2.2$ & $35.1 \pm 2.1$ & $34.1 \pm 1.8$ & 0.59 & 0.12 \\
\hline \multicolumn{7}{|c|}{ Amino acids ${ }^{\mathrm{e}}$ Phloem } \\
\hline Total & $2.49 \pm 0.31$ & $2.89 \pm 0.46$ & $1.96 \pm 0.34$ & $1.86 \pm 0.23$ & 0.42 & $<0.01$ \\
\hline \multicolumn{7}{|c|}{ Glucosinolates $^{\mathrm{f}, \mathrm{g}}$ Leaf } \\
\hline Total & $1.91 \pm 0.34$ & $1.89 \pm 0.25$ & $1.66 \pm 0.37$ & $1.42 \pm 0.17$ & 0.74 & 0.21 \\
\hline IBE & $0.60 \pm 0.12$ & $0.56 \pm 0.10$ & $0.44 \pm 0.11$ & $0.38 \pm 0.07$ & 0.93 & 0.06 \\
\hline PRO & $0.14 \pm 0.04$ & $0.15 \pm 0.03$ & $0.12 \pm 0.05$ & $0.09 \pm 0.02$ & 0.94 & 0.15 \\
\hline RAPH & $0.14 \pm 0.04$ & $0.15 \pm 0.04$ & $0.18 \pm 0.08$ & $0.11 \pm 0.03$ & 0.75 & 0.79 \\
\hline SIN & $0.23 \pm 0.06$ & $0.19 \pm 0.03$ & $0.13 \pm 0.03$ & $0.12 \pm 0.02$ & 0.97 & 0.08 \\
\hline GNA & $0.06 \pm 0.01$ & $0.04 \pm 0.01$ & $0.02 \pm 0.00$ & $0.02 \pm 0.00$ & 0.46 & $<0.01$ \\
\hline TROP & $0.05 \pm 0.01$ & $0.06 \pm 0.01$ & $0.06 \pm 0.00$ & $0.06 \pm 0.00$ & 0.49 & 0.57 \\
\hline $4 \mathrm{OH}$ & $0.12 \pm 0.01$ & $0.14 \pm 0.01$ & $0.14 \pm 0.02$ & $0.13 \pm 0.01$ & 0.34 & 0.58 \\
\hline GBC & $0.49 \pm 0.09$ & $0.43 \pm 0.06$ & $0.44 \pm 0.08$ & $0.39 \pm 0.05$ & 0.60 & 0.73 \\
\hline $4 \mathrm{MeOH}$ GBC & $0.09 \pm 0.01$ & $0.16 \pm 0.02$ & $0.11 \pm 0.03$ & $0.10 \pm 0.01$ & $<0.01$ & 0.41 \\
\hline \multicolumn{7}{|c|}{ Glucosinolates ${ }^{\mathrm{f}}$ Phloem } \\
\hline Total & $0.52 \pm 0.21$ & $1.72 \pm 1.02$ & $0.81 \pm 0.52$ & $1.13 \pm 0.46$ & 0.10 & 0.83 \\
\hline
\end{tabular}

${ }^{a}$ based on linear mixed effects model with main effects Aphids + Nema, no significant interactions, $n=21$ Significant values are indicated in bold (based on False Discovery Rate control, Benjamini and Hochberg 2000). Details of transformation and $t$-values are given in Online Resource 1

${ }^{\mathrm{b}}$ gram dry weight

c percentage

d (\% $\mathrm{N}$ shoot $\mathrm{x}$ shoot biomass)/(\% $\mathrm{N}$ shoot $\mathrm{x}$ shoot biomass $+\% \mathrm{~N}$ root $\mathrm{x}$ root biomass)

e Sugars and amino acids concentrations in the leaf or in phloem $\left(\mu \mathrm{mol} \mathrm{g}{ }^{-1} \mathrm{dw}\right)$

${ }^{\mathrm{f}}$ glucosinolate concentrations in the leaf $\left(\mu \mathrm{mol} \mathrm{g}{ }^{-1} \mathrm{dw}\right)$ and in phloem $\left(\mathrm{nmol} \mathrm{g}{ }^{-1} \mathrm{dw}\right.$ )

$\mathrm{g}$ abbreviations of glucosinolates are listed in Table 1 
verify the outcome of the linear mixed effects model. In all cases, results were congruent with the outcomes from nonparametric tests, and thus only results from the linear mixed effects models (lme) are shown. One phloem sample was lost during processing. Phloem data were analyzed with the same linear mixed effects model (lme) as above, but including harvesting time as covariate. For parameters significantly affected by aphids or nematodes the correlation between numbers per plant and response value was tested with Spearman rank correlation tests.

Aphid numbers per plant over time were used to fit an exponential growth curve and estimate population doubling times. ANCOVAs were used to test whether relations between plant parameters (\%nitrogen in shoot, \%water, and the individual sugars, amino acids, and glucosinolate concentrations) and aphid doubling times interacted with nematode presence. Plants with less than 10 aphid individuals (3 plants with nematodes, 6 plants without nematodes) were omitted from this analysis. To adjust $P$-values to correct for type 1 errors due to multiple testing, sharpened Benjamini and Hochberg (2000) false discovery rate control was performed (Verhoeven et al. 2005) for the lmes and for the ANCOVAs. Significance levels were determined based on the distribution of the $P$-values, and estimates of the true alternative cases and may, therefore, vary between datasets. In Table 2 , individual $P$-values are shown, but only those that were significant after correcting for multiple tests are printed in bold $(P<0.01)$. The multivariate technique partial least squares regression (PLSR) was used to investigate the relation between aphid doubling times and all primary and secondary metabolites of the plant simultaneously. Data were $\log$-transformed, scaled to unit variance, and were meancentered before analyses. The analysis was done for all aphid doubling times and separately for those on plants with nematodes and those on plants without nematodes (3 PLSR in total). The optimal number of latent structures was determined on the basis of "leave one out" validation. Significance of the model was determined by comparing the explained variation in aphid doubling time with the explained variation of 1,000 models using permutations of the aphid doubling time data. A model explaining more variation than $95 \%$ of 1,000 permutations was considered significant. All analyses were done in R 3.0.0 (R Development Core Team 2013) using the 'nlme' and 'pls' packages (Mevik and Wehrens 2007).

\section{Results}

Plant and Leaf Measurements Given the densities used and the duration of the infestation, it was no surprise that the impact of nematodes on plant biomass was more severe than that of aphids. Nematodes significantly decreased shoot biomass on average by $13 \%$, while the effects of aphids on shoot biomass were not significant (Table 2). Root biomass was not significantly affected by either herbivore treatment (Table 2). Aphid population doubling time was significantly increased by nematode presence (Fig. 1a, lme, $d f=13, P<0.01$ ), while nematode numbers were not significantly affected by aphid presence (Fig. 1b). Water percentages in the shoot were significantly lower on plants with nematodes, although the differences were small (Table 2). Nematodes strongly decreased nitrogen allocation to the shoot, resulting in lower nitrogen concentrations (Table 2), while root nitrogen levels increased in plants with nematodes. The C:N ratio in shoots and roots showed the reversed pattern of the nitrogen concentrations, since carbon levels were not affected by nematodes (Table 2). Aphids had no significant effects on nitrogen concentrations or allocation.

Leaf sugar concentrations (glucose) increased in nematodeinfested plants (Table 2). Nematodes decreased arginine, but the total concentration of amino acids was not significantly affected. Aphids did not affect sugar or amino acid levels in the leaves.

Total glucosinolate concentrations in the shoot were not different between treatments, but there were shifts in individual concentrations (Table 2). Gluconapin concentrations in the leaves were reduced by $58 \%$ as a result of nematode infestation, while one minor glucosinolate, gluconapoleiferin, increased in plants with nematodes. In fact, in the absence of nematodes this glucosinolate was detected in only 1 out of 42 plants, while it was found in 41 of the nematode-infested
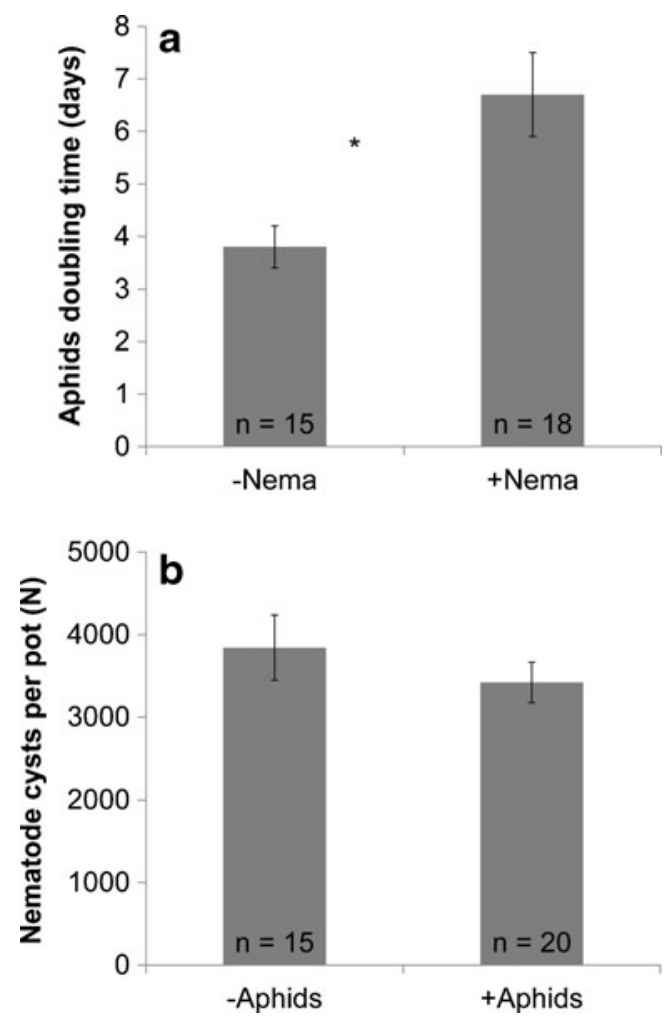

Fig. 1 The presence of nematodes Heterodera schachtii (Nema) increased the doubling time of the aphid Brevicoryne brassicae on Brassica oleracea (a) while the presence of aphids (Aphids) had no significant effect on the number of nematode cysts per pot (b) 
plants. Gluconapoleiferin levels in the shoot were positively correlated with the number of cysts per plant (Fig. 2). Aphids had consistent effects on only one glucosinolate; 4 -methoxyglucobrassicin, which was increased $(+32 \%)$ in the leaves of plants with aphids only. The number of aphids at the end of the experiment correlated positively with 4methoxyglucobrassicin concentrations in the leaves (Fig. 3).

Phloem Measurements Concentrations of compounds in phloem sap varied strongly depending on the harvest time and day (Online Resource 2). This had a large impact on the absolute amounts of the compounds, but not on the detection of treatment effects. The nematode treatments reduced total sugar concentration in the phloem sap by $20 \%$ (Table 2 ), with similar decreases in all individual sugars (data not shown). Four amino acids were detected in all phloem samples: arginine, threonine, leucine, and tyrosine. Aphids alone did not affect total or individual amino acid concentrations (Table 2) in the phloem compared to the control plants, while nematodes reduced total amino acid concentrations by $21 \%$ (Table 2). The individual amino acids showed the same pattern. The concentrations of glucosinolates in the phloem varied widely, from 0.03 to $12.7 \mathrm{nmol} \mathrm{g}^{-1} \mathrm{dw}$. The highest concentration was found in the plant with the highest number of aphids. 4-Methoxyglucobrassicin was found in all phloem samples analyzed, while it was usually one of the less abundant components in the leaves (Table 2). Five other glucosinolates (sinigrin, glucobrassicin, raphanin, gluconapin, and 4-hydroxyglucobrassicin) were found occasionally, in 2$15 \%$ of the phloem samples. There were no significant effects of aphids or nematodes treatments on glucosinolate concentrations in phloem samples.

Linking Aphid Growth Rate to Metabolites Significant effects on aphid population doubling times were found for eight plant parameters. The relation between those plant parameters and

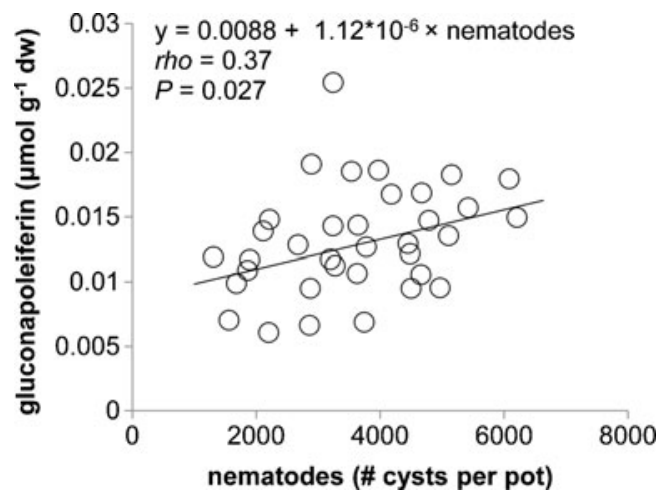

Fig. 2 Spearman Rank Correlation between the number of Heterodera schachtii (cysts per plant) and the concentrations of gluconapoleiferin in the shoot of Brassica oleracea; except for one plant gluconapoleiferin was not detected in the absence of nematodes

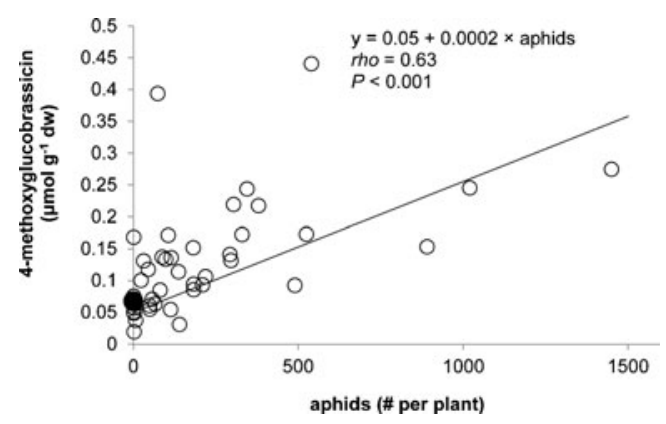

Fig. 3 Spearman Rank Correlation between the number of Brevicoryne brassicae (number of aphids per plant) and the concentrations of 4methoxyglucobrassicin in the shoot of Brassica oleracea; the closed circle denotes the median 4-methoxyglucobrassicin in plants without aphids

aphid population doubling time depended on the presence of nematodes: in the absence of nematodes, aphid population doubling time correlated negatively with both primary and secondary metabolites in the leaves. Three amino acids (arginine, valine, threonine) and five glucosinolates (all aliphatic glucosinolates) correlated negatively with aphid doubling times (Fig. 4a and b, Online Resource 3). However, on plants with nematodes, no significant correlations were found. Similar results were found when exploring the relationship between aphid doubling times and plant parameters with the multivariate partial least squares regression $(P L S R)$. When separate PLSR models were made for the datasets in absence and presence of nematodes, no significant model was found for the aphid doubling times on plants with nematodes. In contrast, in absence of nematodes, $77 \%$ of the variation in aphid doubling times was explained (Online Resource 4) by
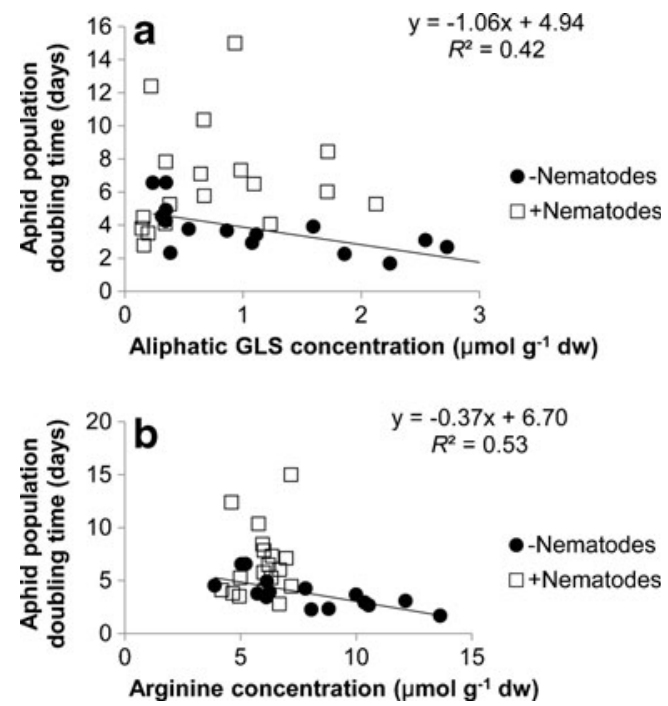

Fig. 4 Pearson Correlation between the doubling time of Brevicoryne brassicae population and a. concentrations of aliphatic glucosinolates or b. arginine in the leaves of Brassica oleracea; closed circles are plants without nematodes, open squares are plants with nematodes. The correlations were only significant for plants without nematodes (closed symbols) and those correlations are indicated here with trendlines 
the same compounds as above: the amino acids arginine, valine, threonine, and the glucosinolates sinigrin, glucoiberin, progoiterin, glucoraphanin, and gluconapin were all negatively correlated with aphid doubling time.

\section{Discussion}

In this study, inoculation with nematodes altered a number of plant parameters, but there was no evidence that those effects were magnified or reduced by the presence of aphids. Based on these results, we reject our first hypothesis that the shared feeding guild of nematodes and aphids leads to non-additive effects on plant parameters. This result is in strong contrast with other studies where the presence of nematodes had different effects on plant parameters when other herbivores or decomposers were present. Wurst et al. (2006) and Lohmann et al. (2009) observed that nematodes increased glucosinolate levels in the plant, but only when earthworms where present. Van Dam et al. (2005) found that aboveground herbivores induced glucosinolates faster on plants with nematodes than on plants without nematodes. Possibly the infestation levels of aphids in our experiment were not sufficient to induce defense mechanisms and/or affect induction by nematodes via crosstalk. However, a recent study (Soler et al. 2012) found that even lower numbers of $B$. brassicae reduced jasmonic acid levels tenfold in Brassica oleracea within 144 h after attack. Similarly, Kutyniok and Müller (2012) demonstrated effects of ten adult aphids on secondary metabolites in Arabidopsis thaliana already 3 days after infestation. Moreover, Kuśnierczyk et al. (2008) found changes in Arabidopsis thaliana glucosinolate levels already two days after infestation with $B$. brassicae. The responses observed in the latter two studies, however, may have been short-term effects caused by the immediate stress of infesting a relatively small plant $(<0.5 \mathrm{~g}$ fresh weight) with ten or more aphids at once. In our experiment, only five aphids were used to start the populations, which developed in a more natural way over 6 weeks' time. Eventually, the infestation levels should have been sufficient to induce plant defense responses, yet plant biomass and primary metabolites were hardly affected by aphid presence. Possibly, plants can compensate for the effects of aphid infestations when populations start with a few individuals. Another possible explanation for the difference in effects between different experiments stems from the order of attack. In our experiment, nematodes were inoculated 4 weeks earlier than the aphids. Timing and order of attackers has been shown to affect defense levels as well as effects on herbivores (Erb et al. 2008, 2011). In a study where Heterodera schachtii and Brevicoryne brassicae were added at the same time to Arabidopsis thaliana, aphids showed strong effects on plant parameters, and their presence reduced nematode numbers (Kutyniok and Müller 2012).
Both shoot and root herbivores induced changes in glucosinolate composition: aphids increased 4-methoxyglucobrassicin and nematodes gluconapoleiferin. This corroborates earlier findings that root induction increases aliphatic, and shoot induction indole glucosinolates (van Dam and Oomen 2008). The positive correlation between aphid numbers and 4-methoxyglucobrassicin levels in the shoot does not reveal, however, which factor is driving the other. The overall increase in 4-methoxyglucobrassicin concentrations compared to plants without aphids suggests that aphids are inducing 4methoxyglucobrassicin production, as has been found earlier for B. oleracea infested with B. brassicae (Khan et al. 2011). An earlier study showed that Myzus persicae also induced 4methoxyglucobrassicin levels in $A$. thaliana, which enhanced resistance to this generalist aphid species (Kim and Jander 2007). It is not known whether 4-methoxyglucobrassicin or its breakdown products (Agerbirk et al. 2009) could affect this specialist aphid, but in a multivariate analysis, Kos et al. (2012) found a positive correlation between 4-methoxyglucobrassicin and aphid adult weight as well as number of offspring.

Nematodes did not affect 4-methoxyglucobrassicin, but changed the levels of several other glucosinolates in the shoots. The increase in gluconapoleiferin was remarkable, since it was essentially not detected in plants without nematodes. The induction of gluconapoleiferin by nematodes was at least 2.5 -fold if we postulate that the concentrations of gluconapoleiferin in plants without nematodes was just below the detection limit. After induction, the levels were still low (min 0.005 max $0.025 \mu \mathrm{mol} \mathrm{g}{ }^{-1} \mathrm{dw}$ ), but it was found consistently in plants with nematodes. We can speculate about the mechanism behind this specific induction. Gluconapoleiferin is the only aliphatic glucosinolate in B. oleracea plant tissues that has five carbon atoms in its side-chain. Sinigrin and glucoiberin have only three (C3), whereas glucoraphanin, gluconapin, and progoitrin have four (C4). Gluconapoleiferin is the hydroxylated product of glucobrassicanapin, the alkenyl product of glucoalyssin, a side chain modification of glucoberteroin (Sun et al. 2011). The precursors could be produced by one more cycle of side chain elongation (Padilla et al. 2007), and the side chain modifications could be performed by the same enzyme converting $\mathrm{C} 3$ and $\mathrm{C} 4$ glucosinolates. However, none of these precursors were found in our samples, and thus the simplest pathway to gluconapoleiferin would be a chain elongation of progoitrin. The biological relevance of the increased levels of gluconapoleiferin also remains unclear. It has been reported to increase susceptibility to the fungus Sclerotina sclerotiorum in Brassica napus (Fan et al. 2008), but nothing is known about effects on aphids. Similarly, it is unclear how the glucosinolate gluconapin, which was decreased by nematodes, affects aphid populations. Other studies that addressed effects of nematodes on leaf glucosinolates (Kabouw et al. 2011; Kutyniok and Müller 2012; Lohmann et al. 2009; Van Dam et al. 2005; Wurst et al. 2006) showed no clear pattern, but we can conclude 
that there was no increase in total glucosinolates after nematode infestation. In terms of specific glucosinolate classes, increases (Lohmann et al. 2009), decreases (van Dam et al. 2005; Wurst et al. 2006), or no effects (Kutyniok and Müller 2012) have been found in aliphatic compounds. Changes in indole glucosinolate concentrations after nematode infestation have not yet been reported.

Our hypothesis that primary metabolites correlate with aphid performance could not be supported. Nematodes affected both primary and secondary metabolites, but we could not correlate those to aphid performance. Decreases in arginine and threonine concentrations also were observed by Bezemer et al. (2005) who found that nematodes decreased amino acid levels in the phloem of grasses. Based on the relationship between arginine concentration and aphid doubling time (Fig. 4b), the average reduction of arginine concentration by nematodes from 8 to $6.2 \mu \mathrm{mol} \mathrm{g}$ $\mathrm{dw}$ in our study could explain an increase in aphid population doubling time from 3.8 to 4.4 days. However, other factors will have played a role as well since aphid population doubling time actually increased to 6.7 days in the presence of nematodes. The negative effects of nematode presence on aphids could be caused by a combination of primary and secondary metabolites as well as by mechanical changes, and hence the precise mechanism may be difficult to reveal. Alternatively, the plant parameter responsible for the effects might not have been included in our measurements, or was measured at an inappropriate time. Induced defense might change over time. Phloem chemistry was expected to correlate with aphid growth rate, but the analysis was hampered by fluctuations in concentrations during the day and between days in this study. Diurnal fluctuations in phloem are somewhat controversial due to methodological issues (Kallarackal et al. 2012), and the present experiment was not aimed at detecting those fluctuations. Regardless of the lack of correlation between measured chemical parameters and aphid numbers, the delay in aphid population growth by nematode presence could be caused by mechanical factors, such as potential changes in cuticular waxes (Kuhlmann and Müller 2010), leaf toughness, or water content. The slight decrease in water content of the shoots caused by nematodes may have reduced aphid population growth, as continuous water stress in general impacts negatively on phloem feeding insects (Huberty and Denno 2004).

In Summary, we showed that nematodes interfere with the interactions between aphids and their host plants, and that this correlates with chemical changes in the host plants. However, the correlations between aphid population doubling time and amino acids or glucosinolates disappear when nematodes are present, and this was confirmed by the multivariate analysis. This shows some similarity with the study from Kutyniok and Müller (2012), who found that early Brevicoryne brassicae infestation had a significant impact on the metabolic profile of Arabidopsis thaliana compared to non-infested control plants, but this was not significant when plants were infested with both Brevicoryne brassicae and Heterodera schachtii. In our study, correlations between aphid performance and plant metabolites were found for metabolites that were significantly affected by nematodes (arginine, threonine, gluconapin) as well as for metabolites not significantly affected by nematodes (valine, glucoiberin, progoitrin, glucoraphanin, sinigrin). The compounds themselves may not be the mechanism by which nematode presence affects aphids, but rather a marker for other chemical changes interfering with aphid metabolism. Future research should not only focus on the concentration of compounds that are significantly changed by nematodes, but also consider the correlation between aphids and (non)chemical plant parameters. Additionally, the roles of primary and secondary metabolites in aphid performance need to be verified in experiments using manipulated plants (Kim and Jander 2007) or diets (Macel et al. 2005) to test whether specific compounds are responsible for reduced aphid performance. While such studies are crucial to prove mechanisms, it is also crucial to use ecological and evolutionary relevant study systems, such as wild cabbages, which are much better defended than cabbage cultivars (Harvey et al. 2011).

Acknowledgments We thank Wiecher Smant, Ciska Raaijmakers, and Roel Wagenaar for practical assistance with the chemical analyses and Jeff Harvey and Martine Kos for critically reading an earlier version of the manuscript. This work was financially supported by the ALW-biodiversity program of the Netherlands Organization for Scientific Research (Project ALWPB/05-02). This is publication 5491 of the Netherlands Institute of Ecology (NIOO-KNAW).

Open Access This article is distributed under the terms of the Creative Commons Attribution License which permits any use, distribution, and reproduction in any medium, provided the original author(s) and the source are credited.

\section{References}

Agerbirk N, de Vos M, Kim JH, Jander G (2009) Indole glucosinolate breakdown and its biological effects. Phytochem Rev 8:101-120

Benjamini Y, Hochberg Y (2000) On the adaptive control of the false discovery rate in multiple testing with independent statistics. J Educ Behav Stat 25:60-83

Bezemer TM, De Deyn GB, Bossinga TM, van Dam NM, Harvey JA, Van der Putten WH (2005) Soil community composition drives aboveground plant-herbivore-parasitoid interactions. Ecol Lett 8: 652-661

Bezemer TM, van Dam NM (2005) Linking aboveground and belowground interactions via induced plant defenses. Trends Ecol Evol 20:617-624

Bidart-Bouzat MG, Kliebenstein DJ (2011) An ecological genomic approach challenging the paradigm of differential plant responses to specialist versus generalist insect herbivores. Oecologia 167:677-689

Buchner R (1987) Approach to determination of HPLC response factors for glucosinolates. In: Wathelet JP (ed) Glucosinolates in rapeseed. Martinus-Nijhoff Publishers, Dordrecht, pp 50-58

Carolan JC, Caragea D, Reardon KT, Mutti NS, Dittmer N, Pappan K, Cui F, Castaneto M, Poulain J, Dossat C, Tagu D, Reese JC, Reeck GR, Wilkinson TL, Edwards OR (2011) Predicted effector 
molecules in the salivary secretome of the pea aphid (Acyrthosiphon pisum ): a dual transcriptomic/proteomic approach. J Proteome Res 10:1505-1518

Cole RA (1997) The relative importance of glucosinolates and amino acids to the development of two aphid pests Brevicoryne brassicae and Myzus persicae on wild and cultivated Brassica species. Entomol Exp Appl 85:121-133

Den Ouden H (1954) Het bieten cysteaaltje en zijn bestrijding. I. Methoden te gebruiken bij het onderzoek naar kunstmatige en natuurlijke lokstoffen. Meded Inst Suikercore Bergen op Zoom 24: $101-120$

Erb M, Robert CAM, Hibbard BE, Turlings TCJ (2011) Sequence of arrival determines plant-mediated interactions between herbivores. J Ecol 99:7-15

Erb M, Ton J, Degenhardt J, Turlings TCJ (2008) Interactions between arthropod-induced aboveground and belowground defenses in plants. Plant Physiol 146:867-874

Fan ZX, Lei WX, Sun XL, Yu B, Wang YZ, Yang GS (2008) The association of Sclerotinia sclerotiorum resistance with glucosinolates in Brassica napus double-low dh population. J Plant Pathol 90:43-48

Girousse C, Bonnemain JL, Delrot S, Bournoville R (1991) Sugar and amino-acid composition of phloem sap of Medicago sativa - a comparative study of 2 collecting methods. Plant Physiol Biochem 29:41-48

Harvey JA, van Dam NM, Raaijmakers CE, Bullock JM, Gols R (2011) Tri-trophic effects of inter- and intra-population variation in defence chemistry of wild cabbage (Brassica oleracea). Oecologia 166: $421-431$

Hbirkou C, Welp G, Rehbein K, Hillnhutter C, Daub M, Oliver MA, Patzold S (2011) The effect of soil heterogeneity on the spatial distribution of Heterodera schachtii within sugar beet fields. Appl Soil Ecol 51:25-34

Heijbroek W, Munning RG, van Swaaij ACPM (2002) The effect of different levels of beet cyst nematodes (Heterodera schachtii) and beet necrotic yellow vein virus (BNYVV) on single and double resistant sugar beet cultivars. Eur J Plant Pathol 108:735-744

Huberty AF, Denno RF (2004) Plant water stress and its consequences for herbivorous insects: a new synthesis. Ecology 85:1383-1398

Hofmann J, Hess PH, Szakasits D, Blöchl A, Wieczorek K, DaxböckHorvath S, Bohlmann H, Van Bel AJE, Grundler FMW (2009) Diversity and activity of sugar transporters in nematode-induced root induced syncytia. J Exp Bot 60:3085-3095

Hol WHG, de Boer W, Termorshuizen A, Meyer KM, Schneider JHM, Van Dam NM, Van Veen JA, Van der Putten WH (2010) Reduction of rare soil microbes modifies plant-herbivores interactions. Ecol Lett 13:292-301

Hong SC, Donaldson J, Gratton C (2010) Soybean cyst nematode effects on soybean aphid preference and performance in the laboratory. Environ Entomol 39:1561-1569

Hong SC, MacGuidwin AE, Gratton C (2011) Soybean aphid and soybean cyst nematode interactions in the field and effects on soybean yield. J Econ Entomol 104:1568-1574

Hopkins RJ, van Dam NM, van Loon JJA (2009) Role of glucosinolates in insect-plant relationships and multitrophic interactions. Annu Rev Entomol 54:57-83

Kabouw P, Kos M, Kleine S, Vockenhuber EA, Van Loon JJA, Van der Putten WH, Van Dam NM, Biere A (2011) Effects of soil organisms on aboveground multitrophic interactions are consistent between plant genotypes mediating the interaction. Entomol Exp Appl 139: 197-206

Kallarackal J, Bauer S, Nowak H, Hajirezaei MR, Komor E (2012) Diurnal changes in assimilate concentrations and fluxes in the phloem of castor bean (Ricinus communis L.) and tansy (Tanacetum vulgare L.). Planta 236:209-223
Kaplan I, Halitschke R, Kessler A, Sardanelli S, Denno RF (2008) Constitutive and induced defenses to herbivory in above- and belowground plant tissues. Ecology 89:392-406

Kaplan I, Sardanelli S, Rehill BJ, Denno RF (2011) Towards a mechanistic understanding of competition in vascular-feeding herbivores: an emperical test of the sink competition hypothesis. Oecologia 166: $627-636$

Khan MAM, Ulrichs C, Mewis I (2011) Water stress alters aphid-induced glucosinolate response in Brassicae oleracea var. italica differently. Chemoecology 21:235-242

Kim JH, Jander G (2007) Myzus persicae (green peach aphid) feeding on Arabidopsis induced the formation of a deterrent indole glucosinolate. Plant J 49:1008-1019

Kos M, Houshyani B, Achhami BB, Wietsma R, Gols R, Weldergergis BT, Kabouw P, Bouwmeester HJ, Vet LEM, Dicke M, Van Loon JJA (2012) Herbivore-mediated effects of glucosinolates on different natural enemies of a specialist aphid. J Chem Ecol 38:100-115

Kuhlmann F, Müller C (2010) UV-B impact on aphid performance by plant quality and plant changes induced by aphids. Plant Biol 12: 676-684

Kuśnierczyk A, Winge P, Jørstad TS, Troczyńska J, Rossiter JT, Bones AM (2008) Towards global understanding of plant defence against aphids - timing and dynamics of early Arabidopsis defence responses to cabbage aphid (Brevicoryne brassicae) attack. Plant Cell Environ 31:1097-1115

Kutyniok M, Müller C (2012) Crosstalk between above- and belowground herbivores is mediated by minute metabolic responses of the host Arabidopsis thaliana. J Exp Bot 63:6199-6210

Lohmann M, Scheu S, Müller C (2009) Decomposers and root feeders interactively affect plant defence in Sinapis alba. Oecologia 160: 289-298

Ludwig-Muller J, Bennett RN, Garcia-Garrido JM, Piche Y, Vierheilig H (2002) Reduced arbuscular mycorrhizal root colonization in Tropaeolum majus and Carica papaya after jasmonic acid application can not be attributed to increased glucosinolate levels. J Plant Physiol 159:517-523

Macel M, Bruinsma M, Dijkstra SM, Ooijendijk T, Niemeyer HM, Klinkhamer PGL (2005) Differences in effects of pyrrolizidine alkaloids on five generalist insect herbivore species. J Chem Ecol 31:1493-1508

McCarville MT, O’Neal M, Tylka GL, Kanobe C, MacIntosh GC (2012) A nematode, fungus, and aphid interact via a shared host plant: implications for soybean management. Entomol Exp Appl 143:55-66

Mevik B, Wehrens R (2007) The pls package: principal component and partial least squares regression in R. J Stat Softw 18:1-24

Mueller MJ, Brodschelm W, Spannagl E, Zenk MH (1993) Signaling in the elicitation process is mediated through the octodecanoid pathway leading to jasmonic acid. Proc Natl Acad Sci U S A 90:7490-7494

Padilla G, Cartea ME, Velasco P, de Haro A, Ordas A (2007) Variation of glucosinolates in vegetable crops of Brassica rapa. Phytochemistry 68:536-545

R Development Core Team (2013) R: A language and environment for statistical computing. R Foundation for statistical computing, Vienna, http://www.R-project.org/

Rossi M, Goggin FL, Milligan SB, Kaloshian I, Ullman DE, Williamson VM (1998) The nematode resistance gene mi of tomato confers resistance against the potato aphid. Proc Natl Acad Sci U S A 95: 9750-9754

Salt DT, Fenwick P, Whittaker JB (1996) Interspecific herbivore interactions in a high $\mathrm{CO}_{2}$ environment: root and shoot aphids feeding on Cardamine. Oikos 77:326-330

Soler R, Badanes-Pérez FR, Broekgaarden C, Zheng S, David A, Boland W, Dicke M (2012) Plant-mediated facilitation between a leaffeeding and phloem-feeding insect in a brassicaceous plant: from insect performance to gene transcription. Funct Ecol 26:156-166 
Sun B, Liu N, Zhao Y, Yan H, Wang Q (2011) Variation of glucosinolates in three edible parts of chinese kale (Brassica alboglabra bailey) varieties. Food Chem 124:941-947

Textor S, Gershenzon J (2009) Herbivore induction of the glucosinolatemyrosinase defense system: major trends, biochemical bases and ecological significance. Phytochem Rev 8:149-170

van Dam NM, Heil M (2011) Multitrophic interactions below and above ground: en route to the next level. J Ecol 99:77-88

van Dam NM, Oomen MWAT (2008) Root and shoot jasmonic acid applications differentially affect leaf chemistry and herbivore growth. Plant Signal Behav 3:91-98

van Dam NM, Raaijmakers CE, van der Putten WH (2005) Root herbivory reduces growth and survival of the shoot feeding specialist Pieris rapae on Brassica nigra. Entomol Exp Appl 115:161-170

van Dam NM, Witjes L, Svatos A (2004) Interactions between aboveground and belowground induction of glucosinolates in two wild Brassica species. New Phytol 161:801-810

van Emden HF, Bashford MA (1971) Performance of Brevicoryne brassicae and Myzus persicae in relation to plant age and leaf amino acids. Entomol Exp Appl 14:349-360
Vandegehuchte ML, De la Peña E, Bonte D (2010) Interactions between root and shoot herbivores of Ammophila arenaria in the laboratory do not translate into correlated abundances in the field. Oikos 119: 1011-1019

Verhoeven K, Simonsen KL, McIntyre LM (2005) Implementing false discovery rate control: Increasing your power. Oikos 108:643-647

Vierheilig H, Bennett R, Kiddle G, Kaldorf M, Ludwig-Muller J (2000) Differences in glucosinolate patterns and arbuscular mycorrhizal status of glucosinolate-containing plant species. New Phytol 146: 343-352

Wardle DA, Yeates GW, Williamson WM, Bonner KI, Barker GM (2004) Linking aboveground and belowground communities: the indirect influcence of aphid species identity and diversity on a three trophic level soil food web. Oikos 107:283-294

Wurst S, Langel R, Rodger S, Scheu S (2006) Effects of belowground biota on primary and secondary metabolites in Brassica oleracea . Chemoecology 16:69-73

Wurst S, van der Putten WH (2007) Root herbivore identity matters in plant-mediated interactions between root and shoot herbivores. Basic Appl Ecol 8:491-499 